\title{
Direct Detection Rates of Neutralino WIMP in MSSM
}

\author{
Yeong Gyun $\operatorname{Kim}^{a}{ }^{1}$ Takeshi Nihei ${ }^{b}$, Leszek Roszkowski ${ }^{a}$, \\ and Roberto Ruiz de Austri ${ }^{c}$
}

${ }^{a}$ Department of Physics, Lancaster University, Lancaster LA1 4YB, England

${ }^{b}$ Department of Physics, College of Science and Technology, Nihon University, Japan

${ }^{c}$ Physics Division, School of Technology, Aristotle University of Thessaloniki, Greece

\begin{abstract}
We present a new analysis of the direct detection rates of the neutralino WIMP in the framework of the minimal supersymmetric standard model. We discuss the impact of the current bounds on the Higgs mass, $B R(b \rightarrow s \gamma),(g-2)_{\mu}$, as well as that of the cosmological range for the neutralino relic abundance. We derive upper and lower limits on the detection rates and compare them with current and planned experimental searches.
\end{abstract}

I'm going to talk about direct detection rates of neutralino WIMP in the minimal supersymmetric standard model. More detailed analysis can be found in [1].

It is well known that the mass density of luminous matter and baryons are much less than the critical mass density. Recent observations show that the energy density of the universe is dominated by the dark energy component and by the non-baryonic dark matter component. A good candidate for the non-baryonic dark matter is present in the spectrum of the minimal supersymmetric standard model with $R_{p}$ conservation. It is the lightest neutralino which is admixture of bino, wino and higgsinos. Because the lightest neutralino is weakly interacting massive, it is expected that the neutralino annihilation cross section gives the relic density with a right order of magnitude.

If the halo of our galaxy consists of neutralino WIMPs, those might be detected by their elastic scattering with terrestrial nuclear target. The MSSM Lagrangian leads to the following low energy effective Lagrangian for neutralino-quark interactions

$$
\mathcal{L}=d_{q} \bar{\chi} \gamma^{\mu} \gamma^{5} \chi \cdot \bar{q} \gamma_{\mu} \gamma^{5} q+f_{q} \bar{\chi} \chi \cdot \bar{q} q+\ldots
$$

Some important features of the scalar (spin independent) interaction are as follows. In most cases, the dominant contribution to the spin-independent amplitude comes from the exchange of the two neutral CP-even Higgs bosons. It is further enhanced when the neutralino is a mixed gaugino-higgsino state. Heavy CP-even Higgs coupling to $d$-type quarks is essentially proportional to $\tan \beta$ for $\tan \beta \gg 1$. And as $m_{H} \simeq m_{A}$ increases, $\sigma_{p}^{S I}$ drops as $m_{H}^{-4}$ because of the $t$-channel propagator effect in $\chi q \rightarrow \chi q$ elastic scattering.

\footnotetext{
${ }^{1}$ Speaker
} 
A convenient quantity which is customarily used in comparing theory and experimental results for SI interactions is the cross section $\sigma_{p}^{S I}$ for WIMP elastic scattering of free proton in the limit of zero momentum transfer:

$$
\sigma_{p}^{S I}=\frac{4}{\pi} \mu_{p}^{2} f_{p}^{2}
$$

where $\mu_{p}=m_{p} m_{\chi} /\left(m_{p}+m_{\chi}\right)$ is the reduced mass of the system. The coefficients $f_{p}$ can be expressed as

$$
\frac{f_{p}}{m_{p}}=\sum_{q=u, d, s} \frac{f_{T q}^{(p)}}{m_{q}} f_{q}+\frac{2}{27} f_{T G}^{(p)} \sum_{c, b, t} \frac{f_{q}}{m_{q}}+\ldots
$$

where $f_{T G}^{(p)}=1-\sum_{q=u, d, s} f_{T q}^{(p)}$, and $f_{T q}^{(p)}$ is given by $<p\left|m_{q} \bar{q} q\right| p>=m_{p} f_{T q}^{(p)}(q=u, d, s)$, and analogously for the neutron. The masses and ratios $B_{q}=\langle p|\bar{q} q| p\rangle$ of light constituent quarks in a nucleon come with some uncertainties. For definiteness, we follow a recent re-evaluation [2] and assume $m_{u} / m_{d}=0.553 \pm 0.043, m_{s} / m_{d}=18.9 \pm 0.8$, and $B_{d} / B_{u}=$ $0.73 \pm 0.02$, as well as

$$
f_{\text {Tu }}^{(p)}=0.020 \pm 0.004, f_{\text {Td }}^{(p)}=0.026 \pm 0.005, f_{T s}^{(p)}=0.118 \pm 0.062 .
$$

For the purpose of our analysis, we take as independent MSSM parameters: the ratio of the neutral Higgs VEV's $\tan \beta$, higgsino mass parameter $\mu$, wino mass $M_{2}$, pseudoscalar Higgs mass $m_{A}$, trilinear couplings $A_{t, b, \tau}$ as well as the soft masses of the sleptons $\widetilde{m}_{\tilde{l}}$ and of the squarks $\widetilde{m}_{\tilde{q}}$. If we assume gauge coupling unification and gaugino mass unification at GUT scale, the relations $M_{1}=\frac{5}{3} \tan ^{2} \theta_{\mathrm{W}} \mathrm{M}_{2}$ hold at weak scale, where $M_{1}$ is the bino mass parameter. We keep this relation and perform a scan over the following parameter range at weak scale.

$$
\begin{aligned}
50 \mathrm{GeV} & \leq M_{2} \leq 2 \mathrm{TeV} \\
50 \mathrm{GeV} & \leq|\mu| \leq 2 \mathrm{TeV}(4 \mathrm{TeV}) \\
50 \mathrm{GeV} & \leq \widetilde{m}_{\widetilde{l}} \leq 2 \mathrm{TeV}(4 \mathrm{TeV}) \\
200 \mathrm{GeV} & \leq \widetilde{m}_{\widetilde{q}} \leq 2 \mathrm{TeV}(4 \mathrm{TeV}) \\
90 \mathrm{GeV} & \leq m_{A} \leq 2 \mathrm{TeV} \\
0 & \leq\left|A_{t, b}\right| \leq 1 \mathrm{TeV} \\
5 & \leq \tan \beta \leq 65
\end{aligned}
$$

where we assume $A_{t}=A_{b}$ and set $A_{\tau}=0$ for simplicity.

In deriving the allowed ranges of $\sigma_{p}^{S I}$ we impose the bounds from colliders and $b \rightarrow s \gamma$ $\left(\mathrm{BR}\left(B \rightarrow X_{s} \gamma\right)=(3.41 \pm 0.67) \times 10^{-4}\right)$ and further required $0.1<\Omega_{\chi} h^{2}<0.2$ for 
neutralino relic density. For the lighter chargino we take $m_{\chi_{1}^{ \pm}}>104 \mathrm{GeV}$, for sleptons the lower limit of $90 \mathrm{GeV}$ and for squarks $200 \mathrm{GeV}$. As regards the lightest Higgs mass $m_{h}$, in much of the parameter space $\left(m_{A}>120 \mathrm{GeV}\right)$ the lower limit on the Standard Model Higgs of $114.1 \mathrm{GeV}$ applies. However there are two important points to note. Firstly, theoretical uncertainties in computing $m_{h}$ in the MSSM are estimated at $2-3 \mathrm{GeV}$. Conservatively, we thus require only $m_{h}>111 \mathrm{GeV}$. Secondly, for $90 \mathrm{GeV}<m_{A}<120 \mathrm{GeV}$, there still remains a sizable range of the $\left(m_{h}, m_{A}\right)$-plane where the lightest Higgs mass given roughly by $m_{h}>0.78\left(m_{A}+21.7 \mathrm{GeV}\right)$ is allowed. We also consider very recent measurement of the anomalous magnetic moment of the muon $a_{\mu}=\left(g_{\mu}-2\right) / 2$ [3], which restricts possible SUSY contribution to $22.7 \times 10^{-10}<a_{\mu}^{\mathrm{SUSY}}<45.1 \times 10^{-10} \quad(1 \sigma)$ with the SM value (the $e^{+} e^{-}$based) obtained in [4].

The allowed ranges of the SI cross section that result from our scans are illustrated in Fig. 1 for $\tan \beta=35, A_{t}=A_{b}=1 \mathrm{TeV}$ and $\mu>0$. In deriving the allowed ranges we have imposed the bounds from colliders and $b \rightarrow s \gamma$, and further required $0.1<\Omega_{\chi} h^{2}<0.2$, as described earlier, but not yet the bound from $(g-2)_{\mu}$.

We can see a big spread of $\sigma_{p}^{S I}$ over some seven (four) orders of magnitude at small (large) $m_{\chi}$. In fact, the upper values of $\sigma_{p}^{S I}$ exceed the latest experimental limits, including the very recent result from Edelweiss [5] and the new limit from the UKDMC Zeplin I detector [6]. Also shown is the CDMS bound and the so-called DAMA region. It is clear that today's experiments have already started probing the most favored ranges of $\sigma_{p}^{S I}$ that come from SUSY predictions for neutralino cold dark matter.

In the four windows of Figs. 2 we show the effect of the most important constraints on the upper and lower limits on the allowed ranges of $\sigma_{p}^{S I}$. Firstly, in the upper left window we show the effect of relaxing the cosmological bound by allowing $\Omega_{\chi} h^{2}<0.1$. Obviously, larger ranges of $\sigma_{p}^{S I}$ now become allowed since an enhancement in the neutralino pairannihilation cross section often, by crossing symmetry, implies an increase in $\sigma_{p}^{S I}$.

In the same window we also show an important effect of including neutralino coannihilation with sleptons (predominantly with the lighter stau) on allowing very low ranges of $\sigma_{p}^{S I}$ at smaller $m_{\chi}$.

In the upper right window of Fig. 2 we present the effect of imposing the constraint from $(g-2)_{\mu}$. We can see that, for this case, $m_{\chi} \lesssim 245 \mathrm{GeV}(1 \sigma \mathrm{CL})$ and $m_{\chi} \lesssim 420 \mathrm{GeV}(2 \sigma \mathrm{CL})$. This upper limit comes from the fact that, as $m_{\chi}$ increases, the SUSY contribution from the $\chi^{0}-\widetilde{\mu}$ and $\chi^{-}-\widetilde{\nu}_{\mu}$ loops become suppressed and at some point becomes too small to explain the apparent discrepancy between the SM and the experimental measurement. On the other hand, the allowed ranges of $\sigma_{p}^{S I}$ are not really affected. 


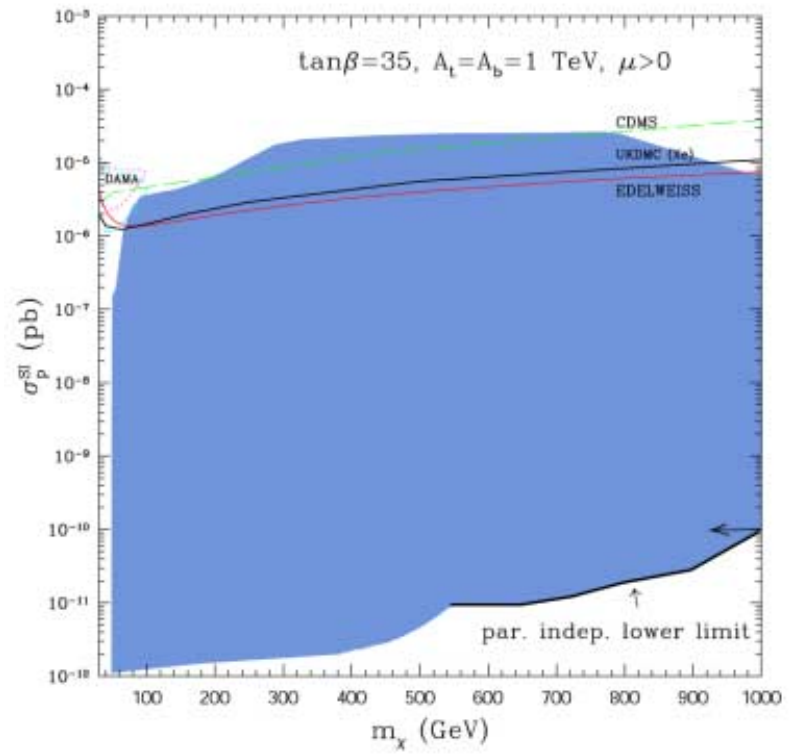

Figure 1: Ranges of $\sigma_{p}^{S I}$ in the general MSSM vs. $m_{\chi}$ for $\tan \beta=35, A_{t}=A_{b}=1 \mathrm{TeV}$ and $\mu>0$, which are allowed by collider bounds, $b \rightarrow s \gamma$ and $0.1<\Omega_{\chi} h^{2}<0.2$, but not from $(g-2)_{\mu}$. Also marked are some results of recent experimental WIMP searches. The thick black line indicates a parameter-independent lower bound at $m_{\chi}>550 \mathrm{GeV}$. No similar bound can be set for lower $m_{\chi}$ because of the neutralino-slepton coannihilation effect.

In the same window we also show the effect of relaxing the constraints from $b \rightarrow s \gamma$. We can see that if it were not imposed, the upper limit on $\sigma_{p}^{S I}$ would significantly increase at large $m_{\chi}$. In this region, the mass of the pseudoscalar Higgs, and therefore also the heavier scalar, is rather small, thus giving larger $\sigma_{p}^{S I}$. However, the mass of the charged Higgs is then also on the lower side, and a cancellation between a charged Higgs-top quark loop and chargino-stop loop contribution is not sufficient to reduce $\operatorname{BR}\left(B \rightarrow X_{s} \gamma\right)$ to agree with the experimental limit.

In the lower left window of Fig. 2 we present the sensitivity of the upper limit on $\sigma_{p}^{S I}$ to the lower limit on the light Higgs mass. We can see that it would sizeably decrease if we neglected the region of small $90 \mathrm{GeV} \lesssim m_{h} \lesssim 111 \mathrm{GeV}$ which is still allowed at $m_{A} \lesssim 120 \mathrm{GeV}$, and instead required $m_{h}>111 \mathrm{GeV}$ for all $m_{A}$. Note also that the new experimental limits are for the most part inconsistent with the possibility of the light Higgs scalar.

In the same window and in the lower right window we explore the existence of the lower limit on $\sigma_{p}^{S I}$ and its dependence on the assumed upper limit on $m_{A}$ and $\mu$, respectively. As we can see, the lowest values of $\sigma_{p}^{S I}$ are often to a large extent determined by a somewhat subjective restrictions from above on these parameters. As one allows either $\mu$ or $m_{A}$ above $1 \mathrm{TeV}$ the lower limit on $\sigma_{p}^{S I}$ relaxes considerably. 

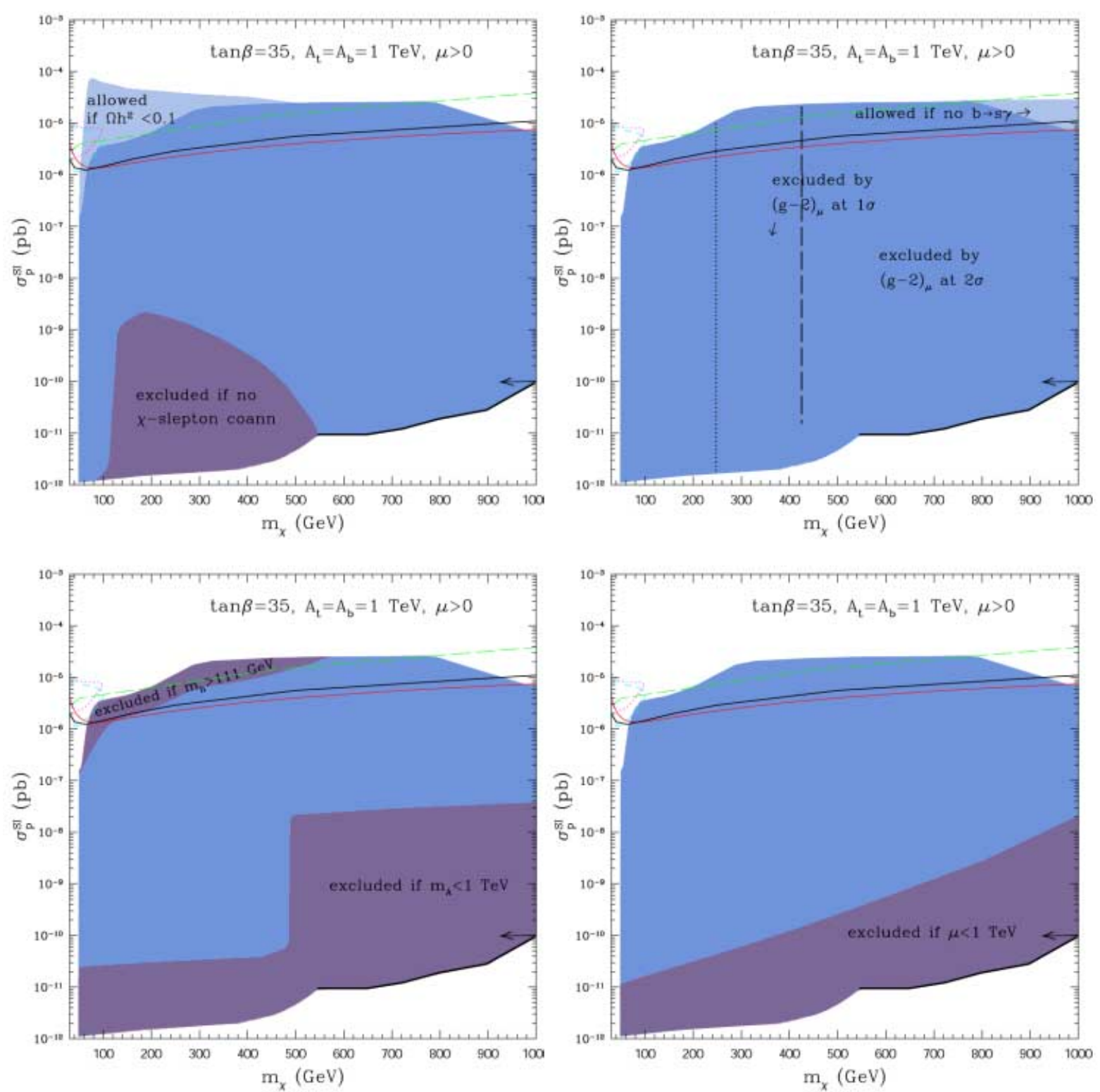

Figure 2: Sensitivity of upper and lower limits on $\sigma_{p}^{S I}$ in Fig. 1 to various assumptions and constraints. Upper left window: the light blue region would be allowed if $\Omega_{\chi} h^{2}<0.1$. The darkred region would be excluded if one neglected the effect of neutralino-slepton coannihilation. Upper right window: the light blue region would be allowed if one lifted the constraint from $b \rightarrow s \gamma$. The regions to the right of the vertical dotted (dashed) lines are excluded by imposing current $1 \sigma(2 \sigma)$ CL bound from $(g-2)_{\mu}$. Lower left window: the upper dark-red region would be excluded by assuming $m_{h}>111 \mathrm{GeV}$ for all $m_{A}$ (i.e., by neglecting a window of lighter $m_{h}$ which is still allowed for $m_{A}<120 \mathrm{GeV}$ ). Also shown in this window is the effect of restricting $m_{A}<1 \mathrm{TeV}$. Lower right window: the same as for $m_{A}$ but for $\mu$. 

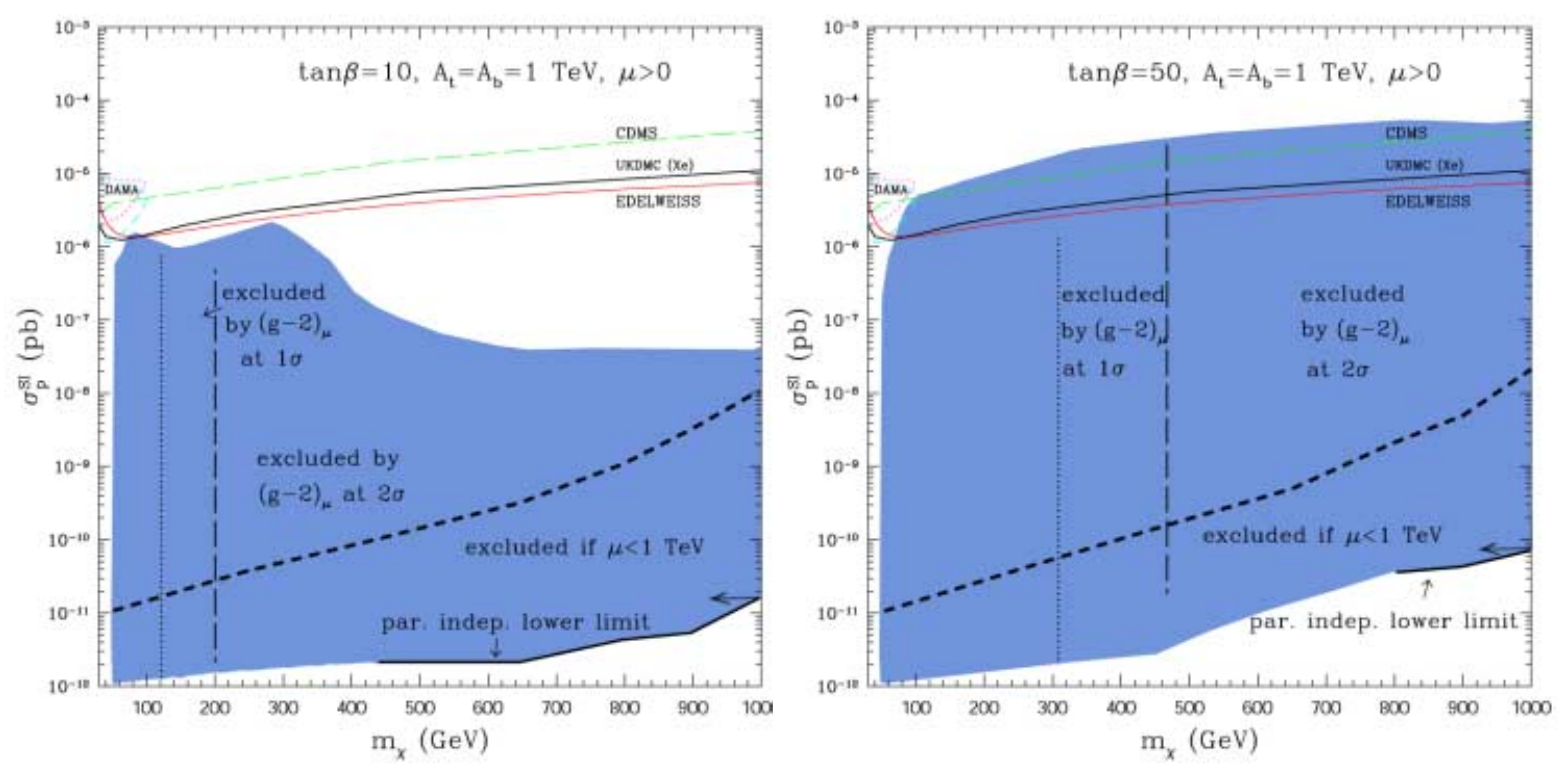

Figure 3: The same as in Fig. 1 but for $\tan \beta=10$ (left window) and 50 (right window). Also marked is the effect of imposing $\mu<1 \mathrm{TeV}$.

In the left and right window of Fig. 3 we present the cases of $\tan \beta=10$ and 50, respectively. Note that, for small $\tan \beta=10$ the largest allowed values of $\sigma_{p}^{S I}$ are roughly an order of magnitude smaller than at $\tan \beta=50$ because of the $\tan \beta$-dependence of the heavy scalar coupling to down-type quarks, as mentioned earlier.

Finally, in Fig. 4 we summarize the results for the full scan conducted so far for $\tan \beta=$ $5,10,35,50,55,60,65$ and for $\mu>0$. We repeat that, in determining the allowed (blue) region we applied the constraints from collider searches, $b \rightarrow s \gamma$ and $0.1<\Omega_{\chi} h^{2}<0.2$. The lower limit on $\sigma_{p}^{S I}$ mostly comes from low $\tan \beta$ and, where possible, we mark with the solid line the parameter-independent lower limit. The effect of restricting $\mu<1 \mathrm{TeV}$ is marked with a dashed line. Also marked is the impact of the new measurement of $(g-2)_{\mu}$ on the mass of the neutralino. If confirmed, this will imply rather stringent upper limits on $m_{\chi}$, i.e, $m_{\chi} \lesssim 350 \mathrm{GeV}(1 \sigma C L), m_{\chi} \lesssim 510 \mathrm{GeV}(2 \sigma C L)$.

We have delineated the ranges of the SI cross section $\sigma_{p}^{S I}$ in the general MSSM, which are consistent with all the current experimental bounds and for which one finds the expected amount of dark matter. We have further discussed the dependence of our results on the underlying theoretical assumptions. While the ranges which we have obtained span over more than six orders of magnitude, we find it encouraging that the experimental sensitivity that has recently been reached, now allows one to explore our theoretical predictions for the MSSM. 


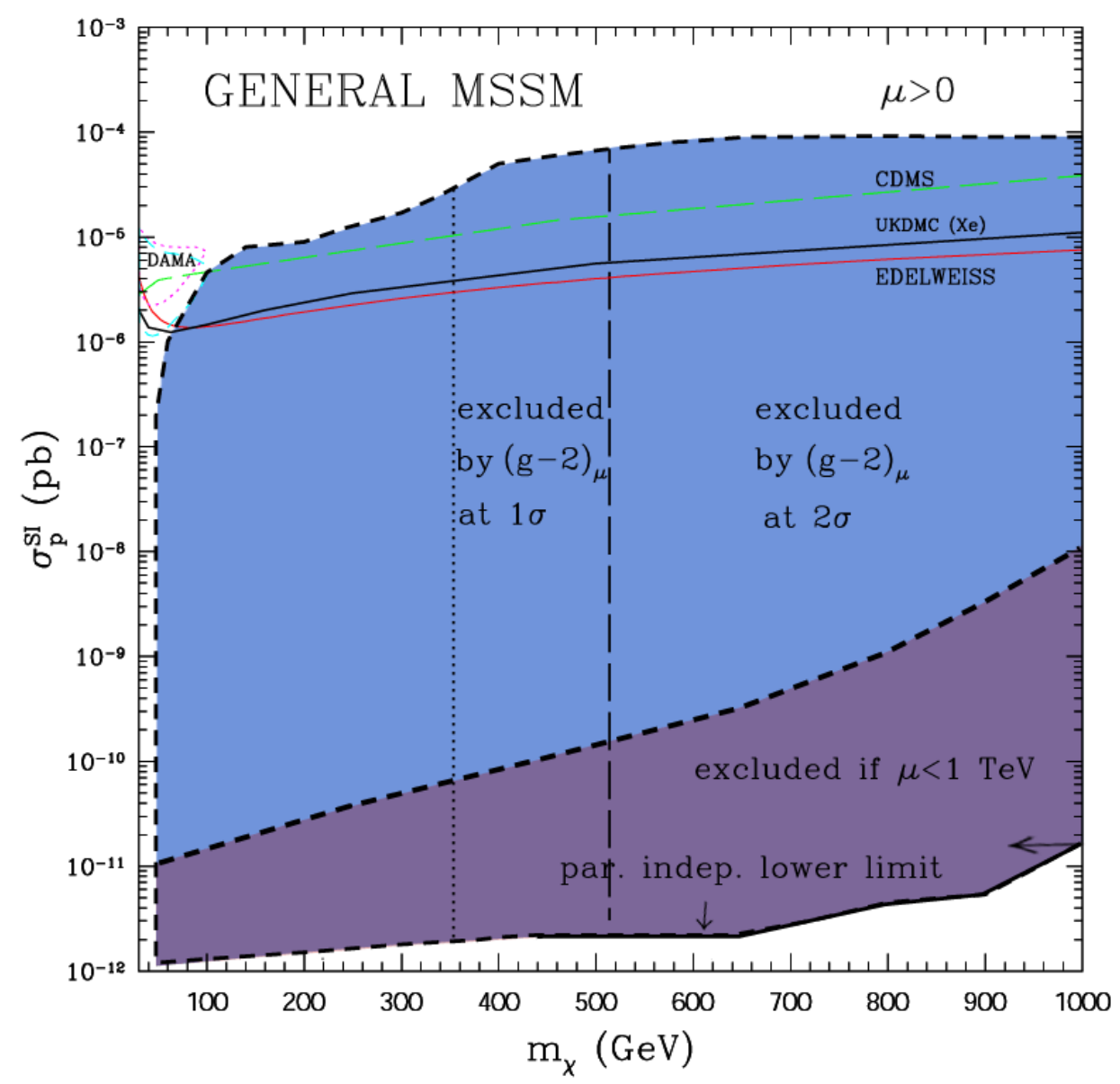

Figure 4: Ranges of $\sigma_{p}^{S I}$ in the general MSSM vs. $m_{\chi}$ for $\mu>0$, which are allowed by collider bounds, $b \rightarrow s \gamma$ and $0.1<\Omega_{\chi} h^{2}<0.2$. Also marked are some results of recent experimental WIMP searches. The thick black line indicates a parameter-independent lower bound. The region below the dashed line is excluded if one imposes the constraint $\mu<1 \mathrm{TeV}$. The ranges of $m_{\chi}$ to the vertical lines are excluded at $1 \sigma$ and $2 \sigma$ CL by the current discrepancy between the experimental value of $(g-2)_{\mu}$ and the Standard Model prediction.

\section{References}

[1] Y.G.Kim, T. Nihei, L.Roszkowski, and R. Ruiz de Austri, hep-ph/0208069.

[2] J. Ellis, A. Ferstl, and K. A. Olive, Phys. Lett. B 481, 304 (2000), hep-ph/0001005.

[3] G.W. Bennet, et al., Muon $g-2$ Collaboration, hep-ex/0208001.

[4] M. Davier, S. Eidelman, A. Hocker, and Z. Zhang, hep-ph/0208177.

[5] A. Benoit et al., EDELWEISS Collaboration, hep-ph/0206271.

[6] N.J.T. Smith, et al., talk at the 4th International Heidelberg Conference On Dark Matter In Astro and Particle Physics, Cape Town, South Africa, 4 - 9 February 2002, to appear in the Proceedings. 